\title{
Young stellar population and star formation history of W4 HII region/Cluster Complex
}

\author{
Neelam Panwar*
}

Department of Physics \& Astrophysics, University of Delhi, Delhi-110007

\begin{abstract}
The HII region/cluster complex has been a subject of numerous investigations to study the feedback effect of massive stars on their surroundings. Massive stars not only alter the morphology of the parental molecular clouds, but also influence star formation, circumstellar disks and the mass function of low-mass stars in their vicinity. However, most of the studies of low-mass stellar content of the HII regions are limited only to the nearby regions. We study the star formation in the W4 HII region using deep optical observations obtained with the archival data from Canada - France Hawaii Telescope, Two-Micron All Sky Survey, Spitzer, Herschel and Chandra. We investigate the spatial distribution of young stellar objects in the region, their association with the remnant molecular clouds, and search for the clustering to establish the sites of recent star formation. Our analysis suggests that the influence of massive stars on circumstellar disks is significant only to their immediate neighborhood. The spatial correlation of the young stars with the distribution of gas and dust of the complex indicate that the clusters would have formed in a large filamentary cloud. The observing facilities at the 3.6-m Devasthal Optical Telescope (DOT), providing high-resolution spectral and imaging capabilities, will fulfill the major objectives in the study of HII regions.
\end{abstract}

\section{Introduction}

Clusters are the dominant mode of star formation in the Galaxy. Massive stars often form in such systems, playing an important role in the evolution of the parental cloud by photoionising and dispersing the cloud through their strong ionising radiation and stellar winds. Hence, HII regions created by the massive stars are excellent sites to study the feedback effects by massive stars. Massive stars may also cause photoevaporation of disks of the young low-mass stars in their vicinity (see e.g. Bally et al. 1998; Balog et al. 2007), thereby playing a key role in shaping the stellar mass function, star formation efficiency and circumstellar disk evolution of the young stars.

The W4 HiI region, located in the Perseus arm of the Galaxy, is one of such sites which has been a subject of investigations for a long time. It consists of many bright-rimmed clouds (BRCs) and globules pointing towards the ionising stars, suggesting that the stellar feedback is playing a key role in altering the morphology and evolution of the region. The region is empowered by the ionizing photons of massive members of the cluster IC 1805, which lies at the center of the HII region. It is located at a moderate distance of $\sim 2 \mathrm{kpc}$ (Massey et al. 1995, Straižys et al. 2013) and the

*neelam_1110@yahoo.co.in

- "First Belgo-Indian Network for Astronomy \& Astrophysics (BINA) workshop", held in Nainital (India), 15-18 November 2016 
cluster IC $1805\left(\alpha_{2000}=02^{h} 33^{m} 22^{s}, \delta_{2000}=61^{\circ} 26^{\prime} 36^{\prime \prime}\right)$ is embedded in a low extinction cloud of mean $\mathrm{E}(B-V) \sim 0.8 \mathrm{mag}$ (Joshi \& Sagar 1983). Though, the population of high- and intermediate-mass stars are studied, the low-mass stellar population remained mostly unexplored. The low-mass stellar population is necessary to understand the nature of the star formation process and the properties of the stellar systems. Recently, Panwar et al. (2017) have studied the young low-mass stellar population of the cluster. However, there is no study on the young stellar population using a homogeneous dataset for the whole HiI region.

In the present work, we study the spatial distribution of young stellar objects (YSOs) in the region, and search for the clustering to establish the sites of recent star formation. We also studied the influence of massive stars on circumstellar disks.

\section{Identification of YSOs}

\subsection{MASS photometry}

YSOs exhibit excess emission in infrared (IR) wavelengths due to their circumstellar disks and therefore occupy distinct positions in IR colour-colour (CC) diagrams due to their different characteristics. Hence, they can be easily identified. The near-infrared (NIR) $(J-H) /\left(H-K_{s}\right) \mathrm{CC}$ diagram for the sources in the HII region is used to select the IR excess sources. We used the $\mathrm{JH} K_{s}$ Two Micron All Sky Survey (2MASS) Point Source Catalog (PSC) (Skrutskie et al. 2006) for the sources having a uncertainty below $0.2 \mathrm{mag}$ in all the three bands to ensure high quality data. We identified young stars according to their location in the CC diagram (for details see Chauhan et al. 2009).

\subsection{Wide Infrared Survey Explorer data}

The Wide-field Infrared Survey Explorer (WISE) space telescope has explored the sky in four wavebands $(3.4,4.6,12$, and $22 \mu \mathrm{m})$ and has been uncovering a population of YSOs hindered in the dense clouds. We have used the WISE preliminary release catalog and considered only those sources which have magnitude uncertainties $\leq 0.2 \mathrm{mag}$ and rejected any source with contamination and confusion flags (cc-flags in the catalog) that include any of "D", " $\mathrm{H}$ ", "O" or "P" in any bands. We merged this catalog to our already-assembled catalog of ancillary data via a strictly position-based search within a $1^{\prime \prime}$ radius. We used the series of colour and magnitude cuts (see Koenig et al. 2012) to remove the contaminants such as background galaxies and nebulosity blobs, as well as to identify and classify the YSOs.

\subsection{Spitzer-IRAC data}

Since, the spatial resolution of the first three WISE bands is $6^{\prime \prime}$ and reaches $12^{\prime \prime}$ at $22 \mu \mathrm{m}$, we have also used the Spitzer-IRAC data from Glimpse-360 (P.I. - B. A. Whitney) in two wavebands (3.6 $\mu \mathrm{m}$ and $4.5 \mu \mathrm{m})$ which were taken during the Spitzer warm mission. We considered sources with photometric uncertainties $\leq 0.2 \mathrm{mag}$ in each band as good detections. We identified the YSOs by combining IRAC (3.6 $\mu \mathrm{m}$ and $4.5 \mu \mathrm{m}$ ) data with 2MASS $\mathrm{H} / K_{s}$ and applying a series of IRAC/2MASS colour criteria developed by Gutermuth et al. (2009). IRAC observations at 3.6, 4.5, 5.8 and $8.0 \mu \mathrm{m}$ wavelengths were also used for the central region. 


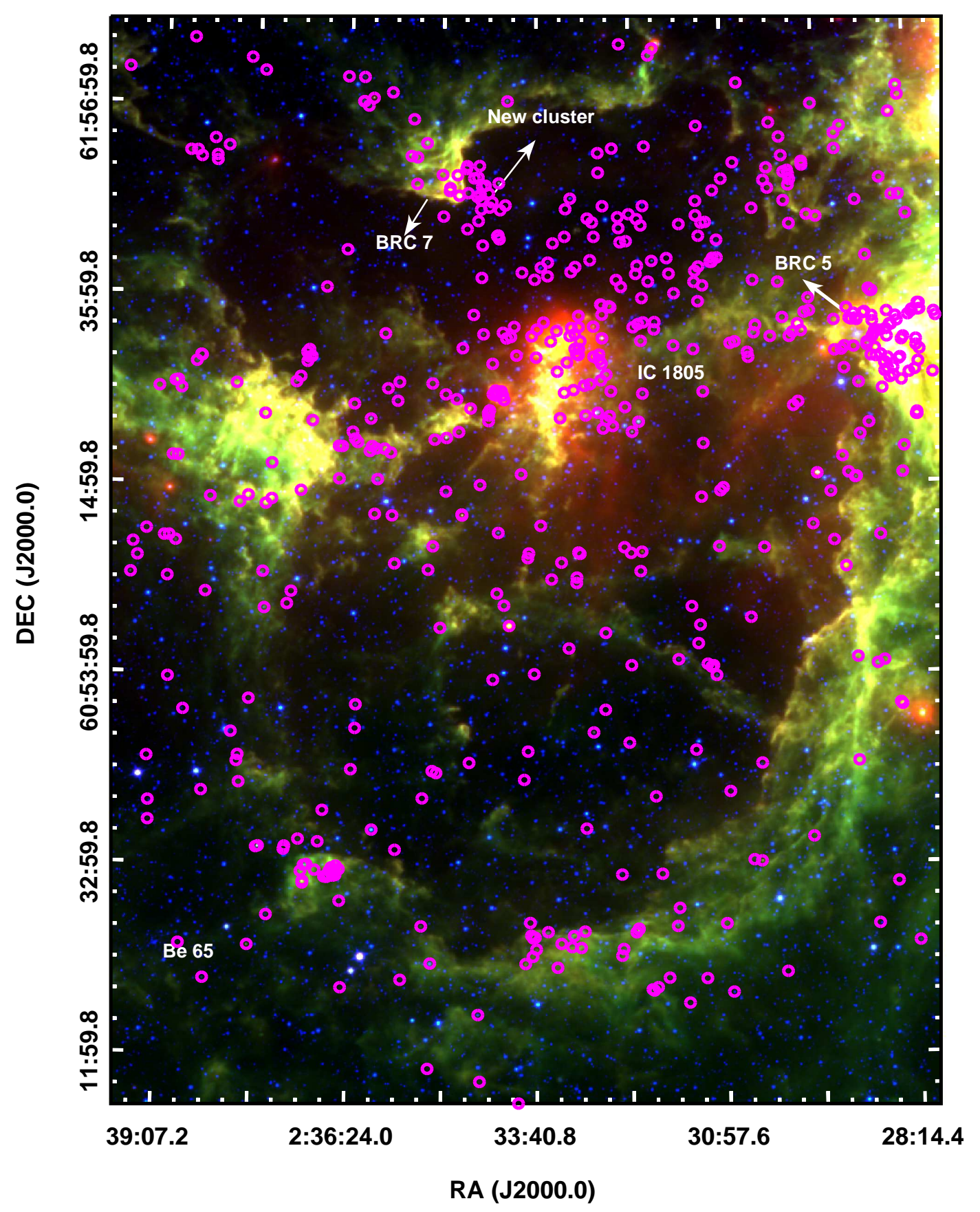

Figure 1: WISE color-composite image (red : $22 \mu \mathrm{m}$; green : $12 \mu \mathrm{m}$; blue : $3.4 \mu \mathrm{m}$ ) of the IC 1805 HII region. Overplotted are the YSOs obtained from 2MASS/IRAC and WISE data. 


\section{Results \& Analysis}

\subsection{Spatial distribution of the YSOs}

We study the spatial distribution of the YSOs obtained from the different datasets. Fig. 1 shows the spatial distribution of the YSOs in the HII region. We can notice an asymmetric distribution of the YSOs. Most of the YSOs are located towards the north-eastern side of the cluster IC 1805. We can also see that most of the YSOs near the BRCs (marked in Fig. 1) are also aligned to the direction of the cluster. For the cluster IC 1805, the Spitzer-IRAC/MIPS and X-ray data from Chandra (Townsley et al. 2014) were available and used to identify the young stars with/without disks (Panwar et al. 2017). To characterise the YSO population of the cluster, we also used the $V, R$ observations from the Canada - France - Hawaii Telescope archive. We derived the age and mass of the young stars using an optical $V$ versus $(V-R)$ colour-magnitude diagram. We studied the influence of massive stars on the circumstellar disk of young stars and found that, although there seems to be a decrease in disk-fraction in the vicinity of massive stars, the variation of disk fraction with respect to the the distance from massive stars is insignificant within the uncertainties. The mid- and far-infrared Spitzer and Herschel images of the region as well as the surface density distribution of the YSOs reveal the filamentary structure of the parental molecular cloud.

\subsection{Clustering in the region : Surface density distribution of stars}

Star clusters often include a distributed stellar population or unrelated background objects. Since clusters are defined as having a density higher than the surrounding field, the methods rely on determining the stellar surface density, the regions above a certain deviation from the background level may be considered as clusters. However, stellar density enhancements can also be due to chance alignment (Froebrich et al. 2008). To study the morphological evolution of the region and find the incidence of clusterings, we generated the isodensity contours for the stars detected in the $2 \mathrm{MASS} \mathrm{K}_{s}$-band.

The surface density distribution reveals, four prominent density peaks in the region. The location of one of the density peaks corresponds to the cluster IC 1805. The second peak is towards the north-east of IC 1805. This density peak matches with the location of the cluster Tombaugh 4 in the literature. It is studied by Subramaniam et al. (2010) in detail who reported it as a relatively old cluster with log age $\sim 8.6-8.7$ located at a distance of $\sim 3.85 \mathrm{kpc}$. In most of the recent studies, the distance of the HII region and the cluster IC 1805 varies from $1.9 \mathrm{kpc}$ to $2.4 \mathrm{kpc}$. Hence Tombaugh 4 seems to be unrelated to the HII region and rather may be a background cluster. The third density peak is towards the south-east of the region. This peak is designated as the open cluster Be 65 in SIMBAD $^{1}$. Moffat \& Vogt (1973) found a distance of $2.2 \mathrm{kpc}$ and reddening E(B-V) of 1.12 mag for this cluster. However, the age of the cluster is estimated as $\sim 10 \mathrm{Myr}$. Hence, this cluster is older compared to the cluster at the center, i.e., IC 1805. The fourth density peak is near the apex of the BRC 7, i.e., towards the northern periphery of the HII region. Panwar et al. (2014) identified several Class II sources near BRC 7, which could be the members of this newly identified cluster. However, to confirm the association of the cluster with the HII region, we will need further investigation.

\section{Conclusion}

The high-resolution spectral/imaging facilities at the 3.6-m Devasthal Optical Telescope (DOT) will be helpful to examine the evolutionary stages of the cluster members and to confirm the connection between the newly identified cluster and W4 HII region/cluster complex.

\footnotetext{
${ }^{1}$ http://simbad.u-strasbg.fr/simbad/
} 


\section{Acknowledgements}

NP is thankful to the anonymous referee for useful comments. NP acknowledges financial support from the Department of Science \& Technology, INDIA, through INSPIRE faculty award IFA-PH-36 at the University of Delhi. This research makes use of data from the Two Micron All Sky Survey (a joint project of the University of Massachusetts and the Infrared Processing and Analysis Center/California Institute of Technology, funded by the National Aeronautics and Space Administration and the National Science Foundation), the Spitzer Space Telescope and the Wide-field Infrared Survey Explorer (operated by the Jet Propulsion Laboratory, California Institute of Technology, under contract with the NASA). This research has made use of the SIMBAD database, operated at CDS, Strasbourg, France.

\section{References}

Bally J., Sutherland R. S., Devine D., Johnstone D. 1998, AJ, 116, 293

Balog Z., Muzerolle J., Rieke G. H. et al. 2007, ApJ, 660, 1532

Chauhan N., Pandey A. K., Ogura K. et al. 2009, MNRAS, 396, 964

Skrutskie M. F., Cutri R. M., Stiening R. et al. 2006, AJ, 131, 1163

Froebrich, D., Meusinger, H., Scholz, A. 2008, MNRAS 390, 1598

Gutermuth R. A., Megeath S. T., Myers P. C. et al. 2009, ApJS, 184, 18

Joshi U. C., Sagar R. 1983, JRASC, 77, 40

Koenig X. P., Leisawitz D. T., Benford D. J. et al. 2012, ApJ, 744, 130

Massey P., Johnson K. E., Degioia-Eastwood K. 1995, ApJ, 454, 151

Moffat A. F. J., Vogt N. 1973, A\&AS, 11, 3

Panwar N., Chen W. P., Pandey A. K. et al. 2014, MNRAS, 443, 1614

Panwar N., Samal M. R., Pandey A. K. et al. 2017, MNRAS, 468, 2684

Straižys V., Boyle R. P., Janusz R., Laugalys V., Kazlauskas A. 2013, A\&A, 554, A3

Subramaniam A., Carraro G., Janes K. A. 2010, MNRAS, 404, 1385

Townsley L. K., Broos P. S., Garmire G. P. et al. 2014, ApJS, 213, 1 